\title{
De interrupciones y sospechas sobre la Antropología filosófica: experiencia para una política de traducción y transformación curricular universitaria en filosofía
}

\section{On interrumptions and suspicions about the philosophical Anthropology: Experience for a policy of translation and transformation of the university curricula in Philosophy}

De interrupções e suspeitas sobre Antropologia filosófica: experiência para uma política de tradução e transformação curricular universitária na filosofia

Dra. Adriana Arpini ${ }^{1}$

Dra. Paula Ripamonti

Recibido: 15/02/2017 - Aceptado: 25/03/2017

\begin{abstract}
Resumen:
El artículo abre una discusión, históricamente situada, acerca del sentido de la Antropología filosófica, su objeto y modos de reflexión y la necesidad de operar sospechas sobre los interrogantes y la trama epistemológica y axiológica subyacente a esta disciplina moderna. Buscamos pensar la relación orgánica de las respuestas antropológicas de la modernidad, en el sentido definido por el peruano Aníbal Quijano y la continuidad del
\end{abstract}

\footnotetext{
1 Argentina, Licenciada, Profesora y Doctora en Filosofía (UNCuyo-Argentina). Se desempeña como investigadora principal de CONICET y docente titular efectiva de "Antropología filosófica" en la Facultad de Filosofía y Letras de la Universidad Nacional de Cuyo. Contacto: aarpini@mendoza-conicet.gob.ar

2 Argentina, Profesora y Doctora en Filosofía (UNCuyo-Argentina), Especialista en Docencia universitaria y postitulada en Investigación educativa (CEA- UNCórdoba). Se desempeña como docente adjunta efectiva de "Antropología filosófica" en la Facultad de Filosofía y Letras de la Universidad Nacional de Cuyo. Contacto: paulafilo70@gmail.com
} 
aparato conceptual de las mismas en las derivas ontológicas del siglo $X X$, para luego proponer interrupciones a la pregunta clásica ¿qué es el hombre?, desde posiciones críticas latinoamericanas (como las de Arturo Roig, Boaventura Santos, Catherine Walsh, entre otros). También, desde la inscripción de esta reflexión en el contexto institucional universitario, pretendemos aportar a actuales reformas curriculares hacia prácticas filosóficas alternativas.

Palabras clave: Antropología filosófica - modernidad - pensamiento latinoamericano - políticas de traducción - ensayo

\section{Abstract:}

The article opens an historical discussion about the meaning of philosophical anthropology, its object and modes of reflection and the need to operate suspicions about questions and the epistemological and axiological plot underlying this modern discipline. We would like to think about the organic relationship of the anthropological responses of modernity, in the sense defined by the Peruvian Aníbal Quijano and the continuity of their conceptual apparatus in the ontological drifts of the Twentieth Century, to then propose interruptions to the classic question, what is Man?, from Latin American critical positions (such as Arturo Roig, Boaventura Santos, Catherine Walsh, among others). Also, from the inscription of this reflection in the university institutional context, we intend to contribute to current curricular reforms towards alternative philosophical practices.

Keywords: Philosophical anthropology - modernity - Latin American thinking - translation policies - essay

\section{Resumo:}

O artigo abre uma discussão, historicamente situada no sentido da Antropologia filosófica, seu objeto e modos de reflexão e a necessidade de operar suspeitas sobre as interrogantes e a trama epistemológica e axiológica subjacente desta disciplina moderna. Procuramos pensar a relação orgânica das respostas antropológicas da modernidade, no sentido definido pelo peruano Aníbal Quijano e a continuidade do aparato conceitual das mesmas nos ângulos ontológicas do século $\mathrm{XX}$, para logo propor interrupções para a clássica pergunta o que é homem? desde posições críticas latino-americanas (como Arturo Roig, Boaventura Santos, Catherine Walsh, entre outros). Assim também, desde o registro desta reflexão no contexto institucional universitário, nosso objetivo é contribuir as atuais reformas curriculares para práticas filosóficas alternativas.

Palavras-chave: Antropologia filosófica - modernidade - pensamento latino-americano - políticas de tradução - ensaio. 


\section{Introducción}

Proponemos una experiencia pedagógica en la forma de una política de traducción y transformación curricular universitaria en filosofía, desde una perspectiva crítica latinoamericana. En las fisuras de una institución que expresa cabalmente una estructura colonial que imbrica formas de saber/poder, desde el espacio curricular de Antropología filosófica, de la carrera de Filosofía de la Universidad Nacional de Cuyo, iniciamos un camino crítico en el que buscamos cuestionar e intervenir prácticas de diálogo, lectura y escritura, que se proponen como formas de racionalidad que soslayan las conflictividades, las operaciones clasificatorias, las funciones ideológicas de los discursos filosóficos y las materialidades vitales.

Por una parte, abrimos una discusión, históricamente situada, acerca del sentido (o sin sentido o nuevos sentidos) de una disciplina como esta, su objeto y modos de reflexión y la necesidad de operar sospechas sobre los interrogantes y la trama epistemológica subyacente a las respuestas antropológicas de la modernidad. Buscamos pensar la relación orgánica de esas respuestas, en el sentido definido por Quijano (1971) y la continuidad del aparato conceptual de las mismas en las derivas ontológicas del siglo XX, para luego proponer interrupciones a la pregunta clásica ¿qué es el hombre? y su trama epistemológica y axiológica binaria ser/tener y aperturas, para descolonizar el campo, desde la tensión eticidad del poder -moralidad de la protesta (Roig 1981 y 2002) y desde el diálogo de saberes (Boaventura Santos 2006 y 2010). Mientras que también, desde la inscripción de esta experiencia en el contexto institucional, buscamos pensar reformas posibles hacia prácticas filosóficas insurgentes de resistir, (re)existir y (re)vivir, que parten de, reconocen y visibilizan, la experiencia como aspecto nodal (Walsh 2013) y se abren a redefinir dónde, para qué y para quién del conocimiento que se pone en circulación y se construye en la universidad (Palermo 2014), en especial en la formación en filosofía. En esta línea, emergen otras formas de traducción, hablamos del ensayo como gesto (Arpini 2003) y como texto genealógico. 


\section{La matriz moderna de la Antropología filosófica}

La configuración de la Antropología filosófica (AF) como disciplina de la Filosofía responde a una matriz moderna tanto en la emergencia de sus interrogantes propios como en los modos en que estos fueron respondidos a lo largo de la tradición occidental. Diferentes filósofos coinciden en situar la problematización de la condición humana en los siglos XV y XVI, con el cambio de paradigma científico geocéntrico, vigente durante unos veinte siglos, en la representación del universo (Groythuesen 1951, García Bacca 1955, Cassirer 1984, Morey 1987, San Martín 1988, Coreth 1991, Buber 1992, entre otros). Esta revolución científica que determinó un fuerte desarrollo de campos como el de la física, orientó interrogantes hacia el lugar que los seres humanos ocupan en el universo, las posibilidades y limitaciones del conocimiento no sólo teórico sino también práctico (ético- político) y metafísico e histórico. Es posible recorrer desde aquí concepciones que revisan la tradición greco-romana y su interpretación medieval reeditando interrogantes de carácter esencialista aunque con articulaciones que podemos denominar modernas y sólo en algunos casos, con líneas de fuga que ponen en cuestión esa tradición.

Así Pico della Mirandola, desde una reinterpretación del relato bíblico de la creación que afirma la libertad como rasgo específico de la naturaleza humana, aboga por respuestas públicas en diálogo con diferentes culturas y hace un llamado a la concordia de los pueblos. Bartolomé de las Casas, por su parte, hace explícita la cuestión antropológica propiamente moderna a partir de la crítica a los métodos e instituciones económicas de la conquista y a su justificación en una supuesta inhumanidad del indio. Desarrolló una antropología de la denuncia que, desde la afirmación de la igualdad del género humano, discute e invierte oposiciones y analogías instituidas (indio = irracional, bárbaro, culpable, etc.; español = racional, cristiano, inocente) y lo hace en el marco de un argumento político que cristaliza cómo los interrogantes se anclan en situaciones vitales que los dotan de sentido.

Pero Pico y Bartolomé no dejan de ser desde el canon filosófico miradas periféricas en la configuración académica europea de la AF. EI 
derrotero pone los ojos en la filosofía cartesiana como fundación de los problemas modernos y con ella en la reafirmación del carácter racional de la naturaleza humana y su escisión respecto de sus alteridades, como el cuerpo y las pasiones. En esta línea, pasando por las versiones políticas de Hobbes y Rousseau o las más fragmentarias de Pascal y Voltaire hasta llegar a Kant, el sujeto humano se define como racional, sin poner en discusión la pretensión universalista que invisibiliza el carácter cultural y racista de las posiciones. La razón se afirma en tensión jerárquica con el cuerpo (siempre masculino, blanco y europeo) y todo lo que este supone. Pero aún más, estas fundaciones antropológicas son ajenas a los acontecimientos, a la historia, a las diferencias tanto dadas como producidas. De aquí que por ejemplo, en Kant, nos encontremos con un nuevo ordenamiento de los saberes, ubicando los argumentos estrictamente filosóficos del lado del sujeto trascendental y los que estima pragmáticos, antropológicos, conjeturales y sin legitimidad universal, del lado del sujeto cultural. Por esto más allá del dualismo operante en sus textos críticos (donde explora la estructuración de una razón pura sin distinciones), las clasificaciones de orden axiológico de sus textos pre-críticos sobre estética y de sus clases de antropología, evidencian el sesgo cultural que atraviesa su obra y muestran los matices de la construcción filosófica moderna europea de la colonización.

Llamamos, así, "matriz moderna" de la AF, como disciplina dentro del campo de la filosofía, a la estructuración de argumentos (eurocentrados), asentados en binarismos con implicaciones antropológicas (como los de pensamiento / naturaleza, razón / cuerpo, ser / tener, cultura / raza, civilizados / salvajes, hombre / mujer, blanco / negro) y posicionamientos epistemológicos en torno de la configuración de saberes acerca de lo humano (homogeneizado) con pretensión de validez universal. Estas implicaciones además se encuentran en tensión con articulaciones políticas concretas, más allá de algunas moderaciones de los discursos de filósofos como Kant o Herder. ${ }^{3}$ 


\section{Críticas eurocentradas a la matriz moderna y lecturas poscoloniales desde América Latina}

Las promesas de la modernidad ilustrada aseguraban que el progreso racional del conocimiento científico permitiría resolver todas las dificultades de la reproducción de la vida, al mismo tiempo que el fortalecimiento de la buena voluntad, apoyada en instituciones que garantizaran un estado de paz duradera, favorecería la plena moralización de los sujetos y el perfeccionamiento de sus disposiciones naturales conforme al ideal de la Humanidad como fin de la historia universal. Aquellas promesas, sin embargo, chocaron con sus propias contradicciones a lo largo del siglo XIX. Karl Marx, Friedrich Nietzsche y Sigmund Freud cuestionaron la capacidad de la razón -o de la conciencia- para superar mediaciones y colocarse en forma directa frente a la verdad. Ellos realizaron el diagnóstico de su propio tiempo señalando las contradicciones que lo atravesaban: capital / trabajo, moral de siervos / moral de señores, eros / thanatos. Cuestionaron la transparencia de la conciencia, minaron la referencia a la razón como condición del conocimiento, la acción y el juicio. Mostraron que más allá de la razón se encuentra el sujeto corporal de necesidades, de valoraciones y deseos. Pusieron en evidencia las mediaciones -trabajo, valores, inconsciente- a través de las cuales se afirma o se niega la vida, se realiza o se aliena, se sublima o se reprime. La dialéctica marxista, la genealogía nietzscheana y el psicoanálisis fueron los métodos a través de los cuales llevaron adelante la crítica, la que, sin embargo, permaneció como crítica inmanente a la modernidad.

Desde una perspectiva antropológica el ejercicio de la sospecha permitió afirmar otras formas de subjetividad constituidas a partir del conflicto y sostenidas en la contradicción: la clase social que no preexiste, sino que se constituye en la lucha de clases (Marx); el sujeto cuya voluntad le permite soportar la intemperie de una cultura decadente y hacerse como niño para crear, jugando, los valores nuevos (Nietzsche); el sujeto deseante que lucha consigo mismo en medio del malestar de la civilización (Freud); el sujeto político que pone la mirada crítica en las instituciones, la erudición y la circulación de los saberes coloniales y denuncia la enajenación epistemológica y cultural 
(Martî). Sin embargo, cabe una pregunta más radical ¿de qué manera estas formas de subjetividad asumen aquello que constituye su propia condición material de posibilidad: la colonialidad del poder?

El pensamiento político, social y filosófico del siglo XX en el periodo que sigue a la Primera Guerra Mundial buscó hacerse cargo de lo acontecido en Europa y el mundo. La condición humana es puesta delante como objeto de preocupación, pero esta vez bajo el prisma de los aportes de Marx, Nietzsche y Freud en tensión con el fracaso del proyecto moderno ilustrado. En este contexto podemos recortar la identificación de modos de racionalidad, su configuración históricocultural, como la distinción de Max Horkheimer entre razón instrumental y razón objetiva y su análisis de los procesos de formalización de los lenguajes como operaciones habilitantes del desarrollo tecno-científico para la guerra y los genocidios; y los análisis políticos de Hannah Arendt en torno de la pérdida de sentido de la acción y la destrucción de los espacios públicos (de la comunidad política) como ámbitos de configuración de la vida humana y de desarrollo y reconocimiento de la pluralidad. En ambos casos, las categorías teóricas emergen de la problematización del tiempo histórico y de acontecimientos como los totalitarismos, por esto encontramos filosofías en las que se juega una crítica a la tradición filosófica y en especial al proyecto ilustrado de progreso de la historia y de emancipación humana, un diagnóstico, en vistas de una comprensión crítico-transformadora del mundo y valoración de la vida humana en su cabal inscripción en la contingencia histórica y ya no en sistemas omnicomprensivos.

Sin duda, es posible establecer otros recorridos, de orden ontológico, como los de Scheler y Heidegger y encontrar búsquedas del sentido de lo humano asentadas en una universalidad cultural que nos pone al habla con categorías como espíritu o lenguaje del ser, sin acuse de recibo respecto de la complejidad y consecuencias del proyecto moderno, económico, político y cultural de la colonialidad.

Desde una perspectiva que exploró posibilidades alternativas, como el existencialismo, por ejemplo, la afirmación de que "la existencia precede a la esencia" de Jean-Paul Sartre pone en cuestión la pretensión de universalidad de la propia subjetividad frente a otras expresiones 
existenciales coloniales; las cuales, sin embargo, quedan finalmente subsumidas como desgarramientos trágicos de una historia (in)capaz de resolver sus contradicciones inmanentes.

Ahora bien, ¿por qué serían necesarios los discursos acerca del hombre? La pregunta de Michel Foucault pone de relieve que el nacimiento de la AF es contemporáneo de un fenómeno que se produce en el campo del saber consistente en que la vida, el trabajo y el lenguaje se presentan como campos empíricos del saber científico, positividades por medio de las cuales es posible determinar la verdad del funcionamiento de lo humano. De modo que saber acerca del hombre es también una manera de ejercer poder, de sujetarlo. Foucault nos obliga a reconocer que sujeto quiere también decir objeto de una sujeción y ofrece una línea de fuga insistiendo en pensar la diferencia, aquello que no puede quedar subsumido conforme a una lógica de la identidad, y señalando incluso la introducción de la raza como criterio para decidir entre lo que se hace vivir o se deja morir ${ }^{4}$. No obstante, no llega a explicar la precedencia de dicho concepto, cuya constitución es consustancial a la configuración de la modernidad.

En efecto, Aníbal Quijano ha señalado que la modernidad comenzó con la constitución de América y del capitalismo colonial/moderno eurocentrado como nuevo patrón de poder mundial, sustentado en dos ejes: uno es "la clasificación social de la población mundial sobre la idea de raza, una construcción mental que expresa la experiencia básica de la dominación colonial y que desde entonces permea las dimensiones más importantes del poder mundial, incluyendo su racionalidad específica, el eurocentrismo" (Quijano 2000 246); el otro es "la articulación de todas las formas histórica de control del trabajo, de sus recursos y productos en torno del capital y del mercado mundial" (Ibid.). La conjunción entre clasificación racial y control de la fuerza de trabajo conformaron el sustrato material posibilitante de la hegemonía centroeuropea sobre las formas de constitución de la subjetividad, de 
la cultura y de la producción del conocimiento; y por tanto también de la Antropología como "disciplina" filosófica.

\section{En torno del sentido actual de la Antropología filosófica. Desafíos para abrir otras políticas de traducción}

Evocando a Gabriel García Márquez y Alejo Carpentier, en 1969, decía Leopoldo Zea que "[a] través de la soledad, de viejos sufrimientos, el latinoamericano (...) se ha encontrado a sí mismo como hombre. El hombre occidental, por su lado, en función de la soledad y el sufrimiento a que sus propias acciones le condujeron, tropezará también con el hombre. (...) un doloroso tropiezo que le hace reconocer en otros rostros, en otras pieles, a hombres no menos hombres que él" (Zea 1969 81). Los acontecimientos del siglo XX habrían hecho conscientes a los intelectuales europeos de las limitaciones de su propia humanidad y de la humanidad de los que consideraban como no iguales. Se trata de un tropiezo que abre miradas no cosificadoras. Los verdaderamente hombres son los diferentes, las víctimas que con su presencia importunan la reflexión ensimismada del hombre occidental, enredada en sus propias creaciones. Zea piensa una integración posible en términos de mestizaje, pero alerta sobre los riesgos de ese desgarro que es el colonialismo interno. Él se hace cargo y explicita la parcialidad y la tragedia del humanismo europeo, de tanta pretensión universal como excluyente, y del humanismo híbrido, fratricida, local, que sólo habla del "Hombre en abstracto", pero se olvida de "los hombres concretos". ${ }^{5}$ Algunas de las inversiones teóricas que propone proceden de lecturas críticas como las de Fanon, desde las que busca interrumpir la continuidad de la colonización, des-enajenarnos, tomar posición, "dejar de ser instrumentos, medio, de otros fines" (Id. 106) y reconocer que estamos ya haciendo historia. De aquí que la función de la filosofía será enfrentar la pregunta por sus propias condiciones en relación con los otros y con las posibilidades de acción, una "filosofía

\footnotetext{
5 Zea 1969 86, citando a Miró Quesada.
} 
sin más del hombre y para el hombre en donde quiera que este se encuentre" (Id. 119).

También desde América Latina Ignacio Ellacuría pregunta ¿a qué se ha debido que los hombres se hayan visto forzados a filosofar? El hombre es la pieza clave de la filosofía y del filosofar, atormentado siempre por la pregunta "qué es realmente la realidad y en ella qué soy realmente como hombre (...). Es aquí donde aparece la historia como lugar de plenificación y de revelación de la realidad: el hombre socialmente considerado y haciendo historia es el lugar de la manifestación de la realidad" (Ellacuría 2001 123). Preguntar por el sentido de las cosas es, en última instancia, una pregunta por el sentido de la vida humana, que involucra tanto la vida personal como la vida social, política e histórica. La filosofía pretende desarrollar a fondo la autonomía, liberando al pensamiento de toda imposición para llevar adelante su tarea de racionalidad crítica, cuyos mecanismos son "la duda y la negación", pues "disminuyen la velocidad de la acción, rompen el carácter monolítico de la organización, dan paso a desahogos individualistas" (Id. 127), al mismo tiempo "muestran la autonomía del pensamiento, su capacidad de convertir la determinación en indeterminación, la necesidad en libertad" (Ibid).

Así pues, la filosofía sirve para saber y para desideologizar. Todos necesitamos de la filosofía, la sociedad la necesita, como saber crítico y creador, capaz de poner en tela de juicio las creencias que perpetúan un orden injusto y violento. Se impone, entonces la pregunta ¿cómo filosofar? Ellacuría señala que el auténtico pensar filosófico surge del examen de la propia situación histórica, para saber cómo es la realidad. De ahí la importancia de enseñar a pensar, de suscitar el espíritu crítico frente a la realidad y a cualquier sistema que intente explicarla, de fomentar la creatividad para el análisis, la comprensión y el hallazgo de respuestas, de formación técnica para entrar en contacto con los grandes pensadores y ser capaces de producir pensamiento propio.

Consecuentes con una tradición de pensamiento crítico latinoamericano, nos parece relevante abrir una discusión, históricamente situada, acerca del sentido o sin sentido, o nuevos sentidos posibles de una disciplina como la AF, su objeto y modos de reflexión y sobre cuáles 
ejes debería articularse una política de traducción de las reflexiones antropológicas.

Retomamos enfoques críticos que, desde la historia de las ideas latinoamericanas, incluyendo las críticas a la colonialidad del poder y del saber, nos ofrecen aperturas para otros "recomienzos", en el sentido de Arturo Roig (1981), de la práctica del filosofar acerca de la vida humana. Esto porque cualquier discurso, oral o escrito, y nuestras prácticas pueden constituirse en archivo, fuentes de un filosofar con vocación crítico-práctica. Los discursos configuran objetivaciones a partir de las cuales mapeamos lo real. De aquí que estos puedan constituirse en formas de opresión o liberación y emancipación, bajo el claro reconocimiento de que "no hay sincronía entre independencia y liberación" (De Oto 2012 s/p). El locus de enunciación de los discursos y la articulación ideológica de los mismos está inscripto siempre en una "sujetividad" concreta, empírica, plural y por esto, producida históricamente. ${ }^{6}$ Una "moral de la emergencia", promovida desde el campo de la acción, forma parte de la historia de América Latina que ha tensionado de diverso modo y con diferentes líneas, la eticidad vigente, pero no ha estado siempre presente de forma sustantiva en el saber universitario, "sino en aquellos intelectuales nuestros, universitarios o no, en los que la originalidad no fue expresamente buscada, sino que fue fruto del encuentro con lo único que nos hace originales, la realidad" (Roig 2002 110). O aquellos pensadores (Mariátegui, Cesaire, Fanon) que, como expresa De Oto, atendiendo a los análisis de la Modernidad en las últimas décadas del siglo XX, muestran la necesidad de leer las subjetividades y la acción histórica en la trama política (y las relaciones sociales) y no por afuera de ella (como ocurre en el caso de las filosofías posmodernas), y "ponían en escena cuánto de aquello que es rechazado del mundo colonial habita en las nuevas formas de protesta y de subjetividad, cuánto se ha negociado en las tramas del significado por medio de resistencias, apropiaciones 0 , simplemente, modos de circulación discursiva, digamos por el propio funcionamiento de los discursos" (De Oto 2012 s/p). 
Entonces, cuando pensamos en revisar críticamente los discursos antropológicos y sus operaciones ideológicas, nos implicamos en procesos de desmontaje de su ontologización y de una universalización que disuelve las marcas históricas de la colonialidad (de clase, de género, de racialización, de cultura) y se desentiende del campo político. Descolonizar el campo epistemológico requiere de un diálogo de saberes, como experiencias de conocimiento, de reconocimiento, de democracia, de trabajo y comunicación y desde aquí articular una "teoría crítica" en la que la realidad se presenta como "un campo de posibilidades", "más allá de lo empíricamente dado", porque atiende a las alternativas frente a lo que resulta criticable ( $y$ en un sentido ético, "indignante") (De Sousa Santos 2006 18).

La inscripción de estos saberes críticos en instituciones de educación superior, nos lleva a una especie de pedagogía de la acción, a reformas posibles hacia prácticas filosóficas que podríamos llamar interculturales, insurgentes, que reconocen los problemas y visibilizan, la experiencia como aspecto nodal, requiriendo "el aprendizaje, desaprendizaje y reaprendizaje, la acción, creación e intervención" (Walsh 2013 24). Discutir con los conceptos heredados, las preguntas impuestas e intervenir el monólogo eurocentrado, para acceder a otras filosofías, otras narraciones, despojadas de sus bases raciales y con esto, retomaríamos la pedagogía "en su aspecto praxístico" (Id. 31), es decir, encarnada en las subjetividades e historias vividas en los territorios, las comunidades, los pueblos, los colectivos.

O en el sentido de Zulma Palermo, nos lleva a repensar las universidad y analizar el modo de desarticular / desprender la colonialidad del saber y su semiosis (habla, escritura y sistemas de signos), desde los "dónde, para qué y para quién del conocimiento que se imparte/construye en ellas", a fin de habilitar una "pluriversalidad" (Palermo 201444 ss). La hipótesis que opera como punto de partida es que las formas de conocer (de anclaje local inicial) tienen la forma de las relaciones de poder y entonces su legitimación, su potencial universalizador y hegemónico, se despliega de forma proporcional al lugar desde el que construye y que se presenta como centro de poder. Una transformación requiere de utopías críticas que apuesten a la reconstrucción de 
la memoria, ampliando el archivo, incluyendo escrituras y oralidades que no soslayen las marcas biográficas de la colonialidad.

Por ello, las políticas de traducción no constituyen formas ajenas ni secundarias ni posteriores de las políticas de formación, de enseñanza y producción de los saberes filosóficos. Estamos pensando no sólo en términos de cuáles rupturas deberíamos operar en torno de las preguntas y respuestas para pensar la vida humana en su complejidad, sino también en los modos de expresión de nuestras reflexiones y sus implicaciones prácticas. Entonces la escritura ${ }^{7}$ asume una dimensión factual o el ámbito de la acción a través de la apertura, por ejemplo, del ensayo como gesto (Arpini 200392 ss) y como texto genealógico. ${ }^{8}$

Como forma discursiva, el ensayo se especifica por su "no-cierre" y su carácter indicativo de un camino a recorrerse en el ámbito de la praxis social, lo propone como "la traducción discursiva del gesto" que "engloba en un mismo espacio, es decir en un mismo texto semiótico, al sujeto, al objeto y a la práctica" (Id. 94). En el ensayo, la gestualidad acompaña la palabra escrita en la medida en que la pone en relación con las prácticas, con las condiciones de su producción, con el contexto histórico, atravesando el entramado textual y direccionando la

7 No sólo la escritura sino también el diálogo y su práctica, como experiencia de pensamiento filosófico, puede asumir la forma de las "comunidades de indagación". Aquí no nos referiremos a ellas especialmente, pero sin duda pueden formar parte de políticas de traducción en el ámbito educativo universitario. Se trata de espacios de encuentro para la circulación de la palabra, motivada en la construcción de un problema filosófico que se busca pensar de forma comunitaria. Esta categoría tiene una larga data en el campo de la denominada "filosofía para niños" de Mathew Lipman y Ann Sharp, pero posee interesantes reformulaciones críticas, teóricas y metodológicas, en América Latina, planteada como "filosofía con niños", en filósofos como Walter Kohan. Una de las cuestiones claves es partir del reconocimiento de que el pensar se articula "en la experiencia, en el encuentro, en la discontinuidad, en el acontecimiento. Toda experiencia, todo encuentro que se precie de tal no puede ser anticipado, previsto, deducido. Es el encuentro con aquello que nos fuerza a pensar, que nos conmueve, que nos deja perplejos, que nos lleva a problematizarnos, a pensar lo que hasta ahora no podíamos pensar. Es la afirmación de lo indeterminado, imprevisto, imprevisible. Donde hay formas, ideas o modelos predeterminados no hay pensamiento" (Kohan 2002 43).

8 Pensamos aquí en el sentido de Nietzsche (1992) y Foucault (1991). 
interpretación. El "ensayo gestual", entonces puede constituirse en una escritura filosófica, es decir, problematizadora, argumentativa, activa, programática, interviniente, que pone en crisis los códigos, las jerarquías y los órdenes y puede forzar su reestructuración desde la interpelación de la facticidad social, que hoy nos reclama el campo de las reflexiones acerca de lo humano. "Una consideración de la gestualidad como producción permitiría un abordaje de todas las prácticas sociales, en particular, aquellas que impliquen un alejamiento y/o modificación de los códigos vigentes" (Ibid.).

En esta línea, el ejercicio ensayístico en el campo filosófico, ese que supone un camino a recorrer, se articula genealógicamente, es decir como escritura que interroga y muestra los comienzos y recomienzos del pensamiento filosófico y las tramas como condiciones de posibilidad histórica, incluyendo otros legados, es decir, haciendo intervenir expresiones gráficas, corporales, testimonios, cartas, otros discursos, jurídicos, educativos, etc. ${ }^{9}$ Se trata de otro modo de vincularnos con la historia: "La genealogía no se opone a la visión de águila y profunda del filósofo en relación a la mirada escrutadora del sabio; se opone, por el contrario al despliegue metahistórico de las significaciones ideales y de los indefinidos teleológicos" (Foucault 1992 8). Por esto, las preguntas genealógicas son aquellas que se desplazan de las formas epistémicas inauguradas desde los qué (esencias) o por qué (causas, origen fundante), para abrirse a los cómo, a los modos, a las maneras, a las configuraciones, a otras verdades. Estas interrogaciones impiden la asimilación o adequatio sujeto-objeto, guardan la distancia necesaria para mostrar el carácter histórico-político de los saberes constituidos, de nuestros modos de relación y de determinación. Podríamos mencionar aquí ese carácter perspectivo al que se refirió Nietzsche y que reseña Foucault como una tarea que entiende la herencia como saber inestable, con pliegues y fisuras y que da especificidad al filo-

9 En otro trabajo, vimos desde Roig, que es necesario desarrollar análisis crítico con los documentos de nuestra historia, tanto de los surgidos desde adentro como externos a los contextos institucionales académicos, y que esto requiere explicitar y desmontar las valoraciones y los andamiajes ideológicos desde los que se posiciona/se produce el sujeto de discurso y se interpreta la historicidad de todo hombre (Ripamonti 2014). 
sofar histórico porque busca procedencias (al mostrar procesos y sus heterogeneidades, sus accidentes, los cuerpos en los se encarna) y emergencias (al mostrar puntos de surgimiento en el marco de un juego de fuerzas) (Foucault 1992 12ss).

Desde una antropología alternativa y descolonizada epistemológicamente, es posible ensayar esas modificaciones en la producción filosófica tanto en contextos educativos como investigativos y académicos. Estos desplazamientos en el campo de una renovada AF, habilitan otros humanismos, diversos, complejos, comprometidos para una reconstrucción de la memoria histórica.

\section{Bibliografía}

Arpini, Adriana. Otros discursos. Mendoza: Qellqaspa, 2003.

Buber, Martín. ¿Qué es el hombre? México: FCE, 1992.

Cassirer, Ernst. Antropología filosófica. México: FCE, 1984.

Coreth, Emerich. ¿Qué es el hombre? Esquema de una antropología filosófica. Barcelona: Herder, 1991.

De Oto, Alejandro. "Siempre se trató de la modernidad y del colonialismo. Una lectura entre teorías coloniales desde una perspectiva fanoniana". CILHA 13 (17) (2012). Recuperado en: http://www.redalyc.org/articulo. oa? id $=181725277012$

De Souza Santos, Boaventura. Conocer desde el sur, para una cultura política emancipatoria. Lima: Fondo Editorial de la Facultad de Ciencias Sociales, 2010.

Ellacuría, Ignacio. Escritos filosóficos III. UCA Editores, San Salvador: Universidad Centroamericana José Simeón Cañas, 2001.

García Bacca, Juan David. Antropología filosófica contemporánea. Barcelona: Anthropos, 1982.

Foucault, Michel. Las palabras y las cosas. Una arqueología de las ciencias humanas. México: Siglo XXI, 1968.

Foucault, Michel. "Nietzsche, la genealogía y la historia". En: Microfísica del poder. Madrid: La Piqueta, 1992, 7-29.

Foucault, Michel. Defender la sociedad. Curso en el Collège de France 1975-76. México: Fondo de Cultura Económica, 2000. 
Kohan, Walter. "Una educación de la filosofía a través de la infancia". Filosofía, narración educación, compiladores Adriana Arpini y Rosa Licata. Mendoza: Qellqaspa, 2002. 43-44.

Lepe-Carrión, Patricio. "Racismo filosófico: el concepto de 'raza' en Immanuel Kant". Revista Filosofía Unisinos 15(1), Jan/apr (2014): 67-83.

Quijano, Aníbal. "Colonialidad del poder, eurocentrismo y América Latina". La colonialidad del saber: eurocentrismo y ciencias sociales. Perspectivas latinoamericanas, compilador Edgardo Lander. Buenos Aires: CLACSO, 2000. Recuperado en en: http://bibliotecavirtual.clacso.org.ar/ar/libros/lander/ quijano.rtf

Morey, Miguel. El hombre como argumento. Barcelona: Anthropos, 1987.

Nietzsche, Friedrich. La genealogía de la moral. Madrid: Alianza Editorial, 1992.

Palermo, Zulma. "La Universidad Latinoamericana en la encrucijada decolonial". Otros logos. Centro de Estudios y Actualización en Pensamiento Político, Decolonialidad e Interculturalidad. Universidad Nacional del Comahue. Año I. No 1, 2014.

Ripamonti, Paula. "Teoría y crítica': claves epistemológicas para un humanismo crítico". Estudios de filosofía práctica e historia de la ideas 16 (1) (2014): 53-61. Recuperado en: http://www.scielo.org.ar/scielo.php?script=sci_artte xt\&pid=S1851-94902014000100006.

Roig, Arturo. Teoría y crítica del pensamiento latinoamericano. México: FCE, 1981.

Roig, Arturo. Ética del poder y moralidad de la protesta. Mendoza: EDIUNC, 2002.

San Martín, Javier. Sentido de la filosofía del hombre. El lugar de la Antropología Filosófica en la Filosofía y en la Ciencia. Barcelona: Anthropos, 1988.

Sartre, Jean-Paul. El existencialismo es un humanismo. Buenos Aires: Huáscar, 1972.

Walsh, Catherine. "Interculturalidad, conocimientos y decolonialidad". Signo y Pensamiento XXIV (46), enero-junio (2005): 39-50.

Walsh, Catherine. "Lo pedagógico y lo decolonial: entretejiendo caminos", editora Catherine Walsh, Pedagogías decoloniales. Querétaro: Cortito que's pa'largo, 2013.

Zea, Leopoldo. "La filosofía occidental tropieza con el hombre". La filosofía americana como filosofía sin más. México: Siglo XXI, 1969. 